\title{
Pre-irradiation Characterization of Radiation Resistant Nanocrystalline and Ultrafine-grained Austenitic Steels
}

\author{
Ryan Carnahan ${ }^{1}$, Andrew Hoffman ${ }^{1}$, Ishtiaque Robin ${ }^{1}$, Malwina Wilding ${ }^{1}$ and Haiming Wen ${ }^{1,2}$ \\ 1. Department of Physics, Nuclear and Electrical Engineering, Idaho State University, Idaho Falls, ID, \\ USA \\ 2. Characterization and Advanced PIE Division, Idaho National Laboratory, Idaho Falls, ID, USA
}

Austenitic steels are important core-internal materials for light-water reactors (LWRs). Life extension of LWRs requires steels with enhanced irradiation tolerance and higher strength. Studies have shown that grain boundaries (GBs) act as sinks for radiation-induced defects [1]; hence, a new generation of radiation tolerant materials may be developed by decreasing grain size and thereby increasing GB volume fraction. The present study involves pre-irradiation characterization of nanocrystalline and ultrafine-grained austenitic steels prepared using two different techniques of severe plastic deformation. The steel samples will then be neutron irradiated at the Advanced Test Reactor located at Idaho National Laboratory.

The austenitic steel samples were processed using two techniques: high-pressure torsion (HPT) and equal channel angular pressing (ECAP) [2]. The processing conditions were: 316 steel processed using ECAP $\mathrm{B}_{\mathrm{c}}$ route for 6 passes at $380{ }^{\circ} \mathrm{C}, 304$ steel processed using ECAP $\mathrm{B}_{\mathrm{c}}$ route for 6 passes at $450{ }^{\circ} \mathrm{C}$, and 304 and 316 steels processed using HPT for 10 turns at $300{ }^{\circ} \mathrm{C}$ under $6 \mathrm{GPa}$ pressure. From transmission electron microscopy (TEM) results, HPT 316 has smaller average grain size $(\sim 91 \mathrm{~nm})$ than the HPT 304 $(\sim 130 \mathrm{~nm})$, and the ECAP 316 has smaller grain size $(\sim 170 \mathrm{~nm})$ than the ECAP $304(\sim 200 \mathrm{~nm})$. Both the HPT and the ECAP samples have significantly increased Vickers micro-hardness compared with the coarse-grained (CG) samples ( 175 HV for CG steels, 350 HV for ECAP 316, 300 HV for ECAP 304, and $\sim 550 \mathrm{HV}$ for the HPT steels).

X-ray diffraction (XRD) was used to study the phase compositions. CG 316 has only austenite phase, and there is not any phase transformation in the material after processing by ECAP or HPT. The phase stability of 316 steel may be due to the higher Ni content than that in 304 steel. CG 304 sample possesses $~ 30 \%$ martensite and $\sim 70 \%$ austenite. ECAP 304 sample exhibited a phase transition to pure austenite. The martensite content in HPT 304 is increased compared to that in CG 304. The micro-strain and accordingly dislocation density in the samples were estimated using the Williamson-Hall method. The estimated dislocation density values are: CG 316 sample $1.4 \times 10^{14} / \mathrm{m}^{2}$, CG 304 sample $2.2 \times 10^{14} / \mathrm{m}^{2}$, ECAP 316 sample $4.8 \times 10^{14} / \mathrm{m}^{2}$, ECAP 304 sample $8.8 \times 10^{14} / \mathrm{m}^{2}$, HPT 316 sample $2.5 \times 10^{15} / \mathrm{m}^{2}$, HPT 304 sample $3.3 \times 10^{15} / \mathrm{m}^{2}$.

TEM analysis of HPT 316 shows smaller equiaxed grains (Figure 1a) and elongated larger grains (Figure 1b). Selected-area electron diffraction (SAED) indicates that the elongated regions tend to contain only face-centered cubic (FCC) austenite. From SAED and converged beam electron diffraction (CBED), the regions with smaller grains contain austenite, body-centered cubic (BCC) ferrite, and cementite $\left(\mathrm{Fe}_{3} \mathrm{C}\right)$. Energy dispersive X-ray spectroscopy (EDS) in scanning transmission electron microscopy (STEM) mode indicates Cr-rich particles in the same region as well. The cementite formation causes carbon-depleted regions to change phase to ferrite, and $\mathrm{Cr}$ also precipitates out. Atom probe tomography (APT) data for HPT 316 shows the formation of carbide particles rich in both Fe and $\mathrm{Cr}$. This is common for cementite or other carbides as $\mathrm{Cr}$ has high solubility in carbides [3]. In comparison, point scans in STEM-EDS did 
not detect second-phase particles in the ECAP 316 sample. The ECAP samples may also have secondphase particles, but it is likely that these particles are larger and not well dispersed. TEM image of HPT 304 with a region with Cr-rich precipitates is shown in Figure 1c, and high-resolution (HR) TEM of a Crrich precipitate in Figure 1d. In general, both HPT 304 and 316 samples exhibit a significant amount of Cr-rich second-phase precipitates (based on STEM-EDS), and TEM and diffraction analysis indicate that these precipitates typically have BCC structure.

Figure 2 shows APT data from the HPT 304 sample, where a significant number of Ni-enriched secondphase particles were observed. Surfaces of these particles are likely preferred nucleation sites for $\mathrm{Cu}$-rich precipitates. Needle-like Cr-rich precipitates, which seem to have grown from one Ni-rich particle to the next, are also seen. From proxigrams, the Cr-rich precipitates are almost pure $\mathrm{Cr}$ at the center of the precipitates, whereas the Ni-rich particles have $\sim 11 \% \mathrm{Ni}$ even at the center of the particles [4].

\section{References:}

[1] Y Chen, et al., Nat. Commun. 6 (2015), p. 7036.

[2] RZ Valiev, RK Islamgaliev, IV Alexandrov, Prog. Mater. Sci. 45 (2000), p. 103.

[3] RC Thomson, HKDH Bhadeshia, Mater. Sci. Tech. 10 (1994), p. 193.

[4] This research is financially supported by U.S. Department of Energy, Office of Nuclear Energy through the NEET-NSUF (Nuclear Energy Enabling Technology - Nuclear Science User Facility) program (award number DE-NE0008524).

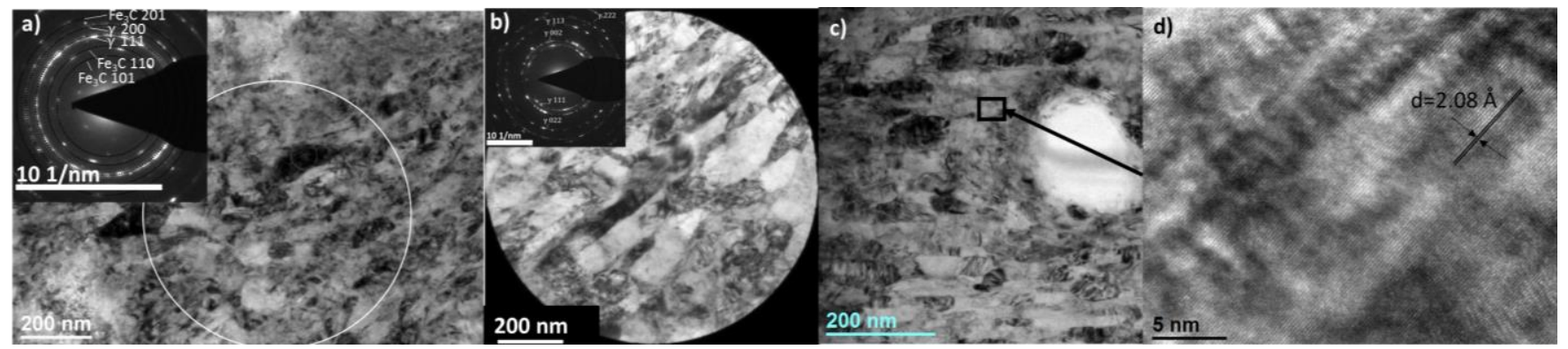

Figure 1. TEM images of HPT 316 showing a) smaller equiaxed grain regions and b) more elongated grain regions with their associated SAED rings. c) TEM image of HPT 304 with Cr-rich precipitates and d) HRTEM image of the highlighted Cr-rich precipitate in (c).
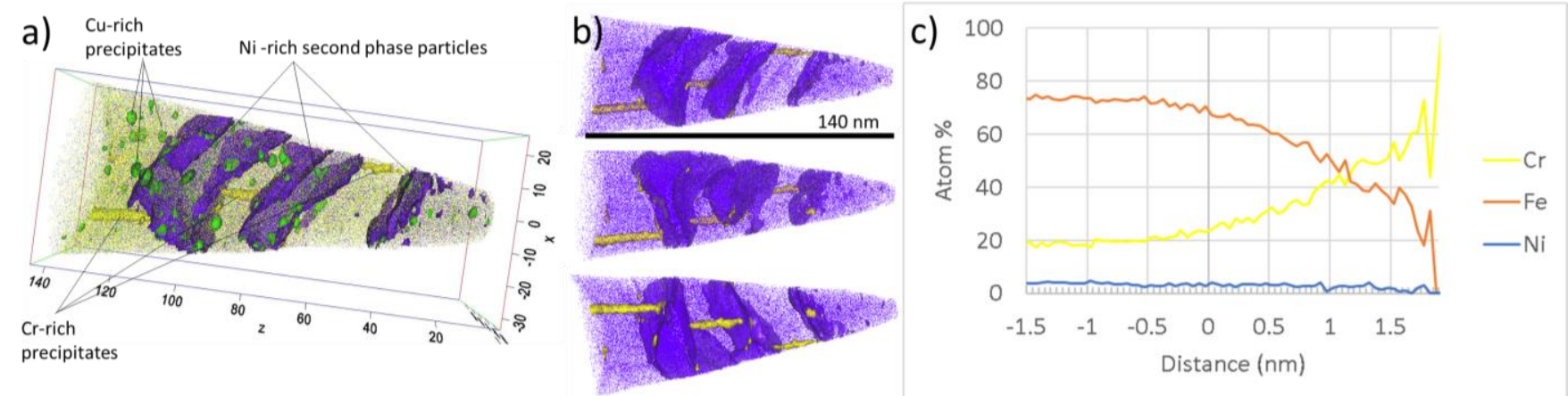

Figure 2. a) APT data for HPT 304, where Ni-rich precipitates are in blue, Cr-rich precipitates in yellow, and $\mathrm{Cu}$-rich precipitates in green. b) View of $\mathrm{Cr}$-rich precipitates from different angles. c) Proxigram of the left Cr-rich precipitate seen in a) and b). 